\title{
Sequestration of latent TGF- $\beta$ binding protein 1 into CADASIL-related Notch3-ECD deposits
}

\author{
Jessica Kast ${ }^{1}$, Patrizia Hanecker ${ }^{1}$, Nathalie Beaufort ${ }^{1}$, Armin Giese ${ }^{3}$, Anne Joutel ${ }^{4,5}$, Martin Dichgans ${ }^{1,2}$, \\ Christian Opherk ${ }^{1 \dagger}$ and Christof Haffner ${ }^{1 * \dagger}$
}

\begin{abstract}
Introduction: Cerebral autosomal dominant arteriopathy with subcortical infarcts and leukoencephalopathy (CADASIL) represents the most common hereditary form of cerebral small vessel disease characterized by early-onset stroke and premature dementia. It is caused by mutations in the transmembrane receptor Notch3, which promote the aggregation and accumulation of the Notch3 extracellular domain (Notch3-ECD) within blood vessel walls. This process is believed to mediate the abnormal recruitment and dysregulation of additional factors including extracellular matrix (ECM) proteins resulting in brain vessel dysfunction. Based on recent evidence indicating a role for the transforming growth factor- $\beta$ (TGF- $\beta$ ) pathway in sporadic and familial small vessel disease we studied fibronectin, fibrillin-1 and latent TGF- $\beta$ binding protein 1 (LTBP-1), three ECM constituents involved in the regulation of TGF- $\beta$ bioavailability, in post-mortem brain tissue from CADASIL patients and control subjects.
\end{abstract}

Results: Fibronectin and fibrillin-1 were found to be enriched in CADASIL vessels without co-localizing with Notch3-ECD deposits, likely as a result of fibrotic processes secondary to aggregate formation. In contrast, LTBP-1 showed both an accumulation and a striking co-localization with Notch3-ECD deposits suggesting specific recruitment into aggregates. We also detected increased levels of the TGF- $\beta$ prodomain (also known as latency-associated peptide, LAP) indicating dysregulation of the TGF- $\beta$ pathway in CADASIL development. In vitro analyses revealed a direct interaction between LTBP-1 and Notch3-ECD and demonstrated a specific co-aggregation of LTBP-1 with mutant Notch3.

Conclusion: We propose LTBP-1 as a novel component of Notch3-ECD deposits and suggest its involvement in pathological processes triggered by Notch3-ECD aggregation.

Keywords: Latent TGF- $\beta$ binding protein 1 (LTBP-1), Small vessel disease, CADASIL, TGF- $\beta$ signaling, Granular osmiophilic material (GOM), Extracellular matrix (ECM)

\section{Introduction}

Cerebral small vessel disease (SVD), a major cause of stroke and vascular dementia, represents the most prevalent neurological disorder in aging societies and thus a considerable health care problem [1,2]. Monogenic forms of SVD such as cerebral autosomal dominant arteriopathy with subcortical infarcts and leukoencephalopathy (CADASIL) are considered valuable model conditions contributing to our understanding of SVD pathomechanisms $[3,4]$. CADASIL vessel pathology shows considerable

\footnotetext{
* Correspondence: Christof.Haffner@med.uni-muenchen.de

${ }^{\dagger}$ Equal contributors

'Institute for Stroke and Dementia Research, Klinikum der Universität München, Ludwig-Maximilians University Munich, Max-Lebsche-Platz 30, 81377 Munich, Germany

Full list of author information is available at the end of the article
}

overlap with sporadic SVD including progressive thickening of the tunica intima and tunica adventitia as well as narrowing of the vascular lumen. These alterations are likely a result of fibrotic events involving increased production of extracellular matrix (ECM) proteins such as collagens $[5,6]$. They are observed primarily in small pial and penetrating arteries, arterioles and capillaries of the brain resulting in reduced cerebral blood flow and white matter damage $[7,8]$.

The gene mutated in CADASIL, NOTCH3, encodes a transmembrane receptor required for arterial differentiation and maturation of vascular smooth muscle cells in small arteries [9]. Pathogenic mutations predominantly affect cysteine residues within individual epidermal growth factor (EGF)-like repeats of the Notch3 extracellular domain (Notch3-ECD) $[10,11]$ leading to Notch3-ECD 
multimerization and accumulation in the tunica media of vessel walls [12]. Notch3-ECD aggregates coincide with large electron-dense deposits known as granular osmiophilic material (GOM), an invariant feature of CADASILaffected vessels [13-15]. The appearance of Notch3-ECD aggregates prior to neurological symptoms in both patients $[16,17]$ and mouse models [18-20] suggest that they represent an early manifestation causative for disease development.

The molecular mechanisms underlying Notch3-ECD deposit formation and the pathological events leading to vessel dysfunction are incompletely understood. A variety of studies using cultured cells or mouse models [19,21-23] have failed to detect alterations in signaling capacity of CADASIL-mutant Notch3, although contradictory results have been reported [24]. Moreover, recently identified patients with hypomorphic NOTCH3 alleles do not show signs of CADASIL [25]. Thus, novel pathogenic roles for mutant Notch3 rather than compromised Notch3 function have been proposed as the primary determinant of the disease [9].

Using scanning for intensely fluorescent targets (SIFT), a confocal technique developed for monitoring protein multimerization in solution [26], we have recently recapitulated the Notch3 aggregation process in vitro and demonstrated its facilitation by CADASIL mutations $[27,28]$. Moreover, we observed co-aggregation of the matricellular protein thrombospondin-2 [28], a known Notch3 interactor and regulator of ECM assembly [29], providing experimental evidence for a pathological coaggregation mechanism. This is supported by recent results obtained from CADASIL brain material enriched for Notch3-ECD deposits by sequential fractionation [30]. Using a mass spectrometry detection approach, a variety of proteins were found to co-fractionate with Notch3-ECD and for two of them, TIMP-3 and vitronectin, disease-related roles were proposed. Notch3-ECD aggregation might thus represent the initiating event of a continuative process involving the recruitment and sequestration of proteins with important roles in normal vessel function.

The dysregulation of the transforming growth factor- $\beta$ (TGF- $\beta$ ) signaling pathway, a key regulator of fibrotic events in various organs including the vasculature [31], has been suggested to contribute to SVD pathogenesis [32]. Moreover, increased TGF- $\beta$ activity has recently been reported in cerebral autosomal recessive arteriopathy with subcortical infarcts and leukoencephalopathy (CARASIL), a recessively inherited SVD syndrome related to CADASIL [33,34]. A specific role of TGF- $\beta$ in CADASIL can be inferred from the fact that fibronectin, fibrillin-1 and members of the latent TGF- $\beta$ binding protein (LTBP) family, important ECM components with a role in TGF- $\beta$ bioactivation [35], were among the factors identified in the proteomic study on CADASIL brains [30]. We now extend this finding using immunohistological approaches on post-mortem brain material and report a dramatic enrichment of fibronectin, fibrillin-1 and LTBP-1 in CADASIL vessels. While fibronectin and fibrillin1 show an immunohistological staining pattern different from Notch3-ECD suggesting accumulation due to fibrotic events secondary to Notch3-ECD aggregation, a striking co-localization with Notch3-ECD deposits was observed for LTBP-1 indicating a role in Notch3-ECD-mediated toxicity. This finding is further supported by analyses demonstrating a direct interaction of LTBP-1 with Notch3ECD and a co-aggregation with mutant Notch3 in vitro.

\section{Materials and methods}

\section{Brain samples and vessel isolation}

Frozen brain samples of five genetically confirmed CADASIL patients (CAD 1-5) and four age- and sexmatched controls with no known cerebrovascular disorders (Ctrl 1-4) as well as paraffin sections of cases 1-3 and controls 1-3, all from the frontal subcortex (see Table 1), were provided by the Brain-Net Biobank (LudwigMaximilians-University Munich) and the CADASIL brain bank (INSERM, Paris) [30]. Their distribution and analysis occurred according to the guidelines of the local ethical committees. Due to limited amounts of obtained material, microvessel isolation could only be performed from CAD 2-5 and Ctrl 1-4 samples. Following a modified version of a previously described protocol [30], $100 \mathrm{mg}$ brain tissue were homogenized in $15 \mathrm{~mL}$ cold Minimum Essential Medium (MEM) (Gibco, Life Technologies) in a glass tissue grinder (Wheaton), homogenates adjusted to a final concentration of $15 \%$ Ficoll and centrifuged at $6000 \mathrm{~g}$ and $4^{\circ} \mathrm{C}$ for $20 \mathrm{~min}$. The pelleted vessels were resuspended in phosphate-buffered saline (PBS)/1\% BSA and transferred onto a 40- $\mu \mathrm{m}$ nylon mesh (Corning Life Sciences). After extensive washing with $\mathrm{PBS}$, isolated vessels were collected in a $50-\mathrm{mL}$ tube and pelleted for $5 \mathrm{~min}$ at $3000 \mathrm{~g}$. The purity of the vessels was verified using a phasecontrast microscope.

\section{Immunohistological analysis}

For immunofluorescence analysis of CAD 1-3 and Ctrl 1-3 samples, frozen $10-\mu \mathrm{m}$ tissue sections were thawed to room temperature and fixed for $15 \mathrm{~min}$ in $4 \%$ paraformaldehyde (PFA) (Morphisto), blocked with PBS/5\% donkey serum (Jackson ImmunoResearch) for $40 \mathrm{~min}$ and probed with primary antibodies in $\mathrm{PBS} / 1 \%$ donkey serum overnight at $4^{\circ} \mathrm{C}$. For detection, Alexa Flour 488-, Cy3- or rhodamine-coupled secondary antibodies (Jackson ImmunoResearch, 1:200) were applied for $1 \mathrm{~h}$ at room temperature. After mounting in ProLong Gold antifade reagent (Life Technologies), slides were examined using an inverse (Axiovert 200 M, Zeiss equipped with AxioCam 
Table 1 Overview of autopsy cases

\begin{tabular}{lllll}
\hline Description & Sex & Age & NOTCH3 mutation & Applications \\
\hline CAD 1 & M & 64 & R110C & Histological stainings \\
CAD 2 & F & 66 & D239_D253del & Histological stainings, Western Blot \\
CAD 3 & M & 68 & C144S & Histological stainings, Western Blot \\
CAD 4 & F & 60 & R153C & Western Blot \\
CAD 5 & F & 70 & C1261R & Western Blot \\
Ctrl 1 & M & 61 & - & Histological stainings, Western Blot \\
Ctrl 2 & F & 55 & - & Histological stainings, Western Blot \\
Ctrl 3 & F & 60 & - & Histological stainings, Western Blot \\
Ctrl 4 & F & 73 & - & Western Blot \\
\hline
\end{tabular}

CAD: CADASIL patient; Ctrl: control patient. Applications were limited by sample amount and type (frozen or paraffin, see Material and methods).

and AxioVision software) or confocal (TCS SP5, Leica equipped with LAS LF software) microscope.

For immunohistochemical analysis of CAD 1-3 and Ctrl 1-3 samples, 8- $\mu \mathrm{m}$ paraffin sections were deparaffinized, rehydrated and antigens unmasked by boiling in $10 \mathrm{mM}$ citrate buffer, $\mathrm{pH} 6.0$ for $30 \mathrm{~min}$. Blocking was performed for $1 \mathrm{~h}$ in $\mathrm{PBS} / 5 \%$ donkey serum and primary antibodies incubated in PBS/1\% donkey serum overnight at $4^{\circ} \mathrm{C}$. After three washes in PBS and blocking of endogenous peroxidases with $0.3 \%$ hydrogen peroxide for $15 \mathrm{~min}$, appropriate biotinylated secondary antibodies (DAKO or Vector laboratories, 1:200) were applied for $1 \mathrm{~h}$ at room temperature. Development was performed with avidin/biotin-horseradish peroxidase (HRP) complex (Vectastain ABC-HRP kit, Vector Laboratories) and 3-amino-9-ethylcarbazole (AEC Peroxidase substrate Kit, Vector Laboratories). To provide cytological detail, sections were counterstained with Mayer's hematoxylin Solution (Sigma) for $1 \mathrm{~min}$ and rinsed in tap water for $10 \mathrm{~min}$ prior to mounting with ProLong Gold. Sections were examined using the Axio Imager M2 microscope (Zeiss).

The following primary antibodies were used: goat polyclonal anti-collagen type IV (Southern Biotech, 1:500), rabbit polyclonal anti-fibrillin-1 (kind gift of Lynn Sakai, Shriners Research Center, Portland [36], 1:100), mouse monoclonal anti-LTBP-1 (MAB388, R\&D Systems, 1:50), mouse monoclonal anti-fibronectin (F3648 Sigma, 1:300), goat polyclonal anti-LAP (AF-246, R\&D Systems, 1:50) or rat monoclonal anti-Notch3-ECD (clone 2G8, raised against the peptide $\mathrm{NH}_{2}$-RSFPGSPPGASNASC-COOH, kind gift of Elisabeth Kremmer, Helmholtz Center, Munich, 1:10).

\section{Sequential protein extraction}

Protein extraction from purified brain vessels was performed by modifying a previously published protocol [30]. Vessels were homogenized in $50 \mathrm{mM}$ Tris- $\mathrm{HCl}$, $\mathrm{pH} 7.5$ using a TissueLyzer (Qiagen) with 5 -mm beads for $3 \mathrm{~min}$ at $50 \mathrm{~Hz}$. After clearance by centrifugation (5 min, 16,300 g), the Tris fraction was collected and the pellet resuspended in $50 \mathrm{mM}$ Tris- $\mathrm{HCl}, \mathrm{pH} 7.5,1 \%$ SDS for $30 \mathrm{~min}$ on ice. Centrifugation for $30 \mathrm{~min}$ at 16,300 g yielded the SDS fraction and a pellet, which was resuspended in $50 \mathrm{mM}$ Tris- $\mathrm{HCl}, \mathrm{pH} 7.5,1 \%$ SDS, $5 \% \beta-$ mercaptoethanol ( $\beta$-ME) for $30 \mathrm{~min}$ on ice while passing through a 29 -gauge needle 5 times. The $\beta$-ME fraction was obtained by centrifugation for $30 \mathrm{~min}$ at $16,300 \mathrm{~g}$. Protease inhibitors (complete Mini EDTA-free, Roche) were used throughout the extraction procedure.

\section{Plasmids}

The short human isoform of LTBP-1 (LTBP-1S) consisting of 1395 amino acids (aa) was used throughout the study. Constructs encoding full-length LTBP-1S and an Nterminally truncated variant encoding aa 528-1395 with a carboxy-terminal hemagglutinin (HA) tag (LTBP-1 $\Delta \mathrm{N}_{-}$ HA) [37] were kindly provided by Jorma Keski-Oja (University of Helsinki, Finland). Full-length LTBP-1S was subcloned into the pEAK-12 vector (provided by Stefan Lichtenthaler, DZNE, Munich) to harbour the CD5 signal peptide, an N-terminal HA tag and a C-terminal V5-His tag. LTBP- $1 \Delta \mathrm{N}$ containing a C-terminal V5-His tag (LTBP$\left.1 \Delta \mathrm{N}_{-} \mathrm{V} 5\right)$ was generated from the full-length clone and LTBP-1 $\Delta$ N_HA by restriction fragment cloning. A Cterminally truncated LTBP-1 variant encoding aa 1-689 and a C-terminal V5-His tag (LTBP-1 $\Delta \mathrm{C}$-V5) in the pTT5 vector [28] was constructed from full-length LTBP-1 by restriction fragment cloning. The N3EGF1-5 wild-type (WT) and N3EGF1-5 C183R constructs used previously [28] were modified to contain a C-terminal Halo tag (Promega).

\section{Cell transfection}

Human embryonic kidney (HEK) 293T cells were grown in Dulbecco's Modified Eagle's Medium (DMEM)-GlutaMAX supplemented with $10 \%$ fetal calf serum and Penicillin/ Streptomycin (all from Gibco, Life Technologies). Smallscale transfections were carried out in 24-well plates using $250 \mathrm{ng}$ plasmid and Fugene (Roche) according to the manufacturer's protocol and serum-free conditioned 
supernatant was collected after $48 \mathrm{~h}$ and cleared by centrifugation. The large-scale transfection procedure used for protein purification from HEK293E cells has been described earlier [28].

\section{Protein purification}

Purification of His-tagged proteins (LTBP-1 $1 \Delta \mathrm{C}_{-} \mathrm{V} 5$ and

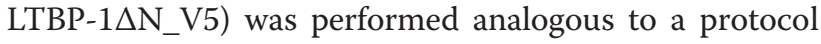
previously described [28]. Purification of N3-EGF1-5 using the Halo System (Promega) will be described elsewhere. Eluted fractions were analyzed by SDS-PAGE followed by silver staining to verify the protein purity. Protein concentrations were measured using the Qubit protein assay (Life Technologies) or BCA Protein Assay Kit (Pierce, Thermo Scientific) according to the manufacturer's instructions.

\section{SDS-PAGE, Western blot and silver staining}

Proteins were separated on polyacrylamide gels (Minigel system, BioRad) and either transferred onto ImmobilonP Membrane (Millipore) using the semi-dry system (Trans-Blot, BioRad) or processed for silver staining (Roti-Black Kit, Roth). For Western blotting, membranes were blocked with Tris-buffered saline (TBS)-0.05\% Tween/4\% skim milk for $1 \mathrm{~h}$ and probed with the following primary antibodies in TBS-0.05\% Tween/4\% skim milk overnight: mouse monoclonal anti-V5 (R960, Invitrogen, 1:1000), rat monoclonal anti-Notch3 ECD (clone 3G6, [28] 1:20), goat polyclonal anti-LAP (AF-246-NA, R\&D Systems, 1:500), mouse monoclonal anti-LTBP-1 (MAB388, R\&D Systems, 1:500) and mouse monoclonal anti- $\beta$-Tubulin (clone TUB 2.1, Sigma, 1:1000). Detection was carried out using HRP-coupled secondary antibodies (DAKO), chemiluminescence development (Immobilon Western HRP Substrate, Millipore) and the Fusion FX7 imaging system (Vilber Lourmat).

\section{Solid-phase binding assay}

$1 \mu \mathrm{g}$ recombinant human Notch3 encompassing aa 40467 (N3EGF1-11-Fc) and IgG $_{1}$-Fc (both R\&D Systems) were dissolved in PBS and coated onto Maxisorp 96-well plates (Nunc) overnight at $4^{\circ} \mathrm{C}$. After blocking with PBS/ $1 \%$ BSA for $1 \mathrm{~h}, 50 \mu \mathrm{L}$ conditioned supernatant from HEK293T cells transfected with LTBP-1, LTBP-1 $\Delta$ C_V5, LTBP- $1 \Delta$ N_HA or $100 \mathrm{ng}$ purified LTBP-1 $\Delta \mathrm{C}_{-} \mathrm{V} 5$ in PBS were allowed to bind for $1 \mathrm{~h}$ at room temperature. Supernatant of mock-transfected cells or PBS was used as a negative control. After washing, detection was performed for $1 \mathrm{~h}$ using the following antibodies: mouse monoclonal anti-V5 (R960, Invitrogen, 1:500) for LTBP1 and LTBP-1 $\triangle$ C_V5; mouse monoclonal anti-LTBP-1 (MAB388, R\&D Systems, 1:500) for LTBP-1 $\Delta$ N_HA. Following incubation with anti-mouse-HRP (DAKO, 1:1000) for $1 \mathrm{~h}$, immune complexes were visualized by TMB
Microwell Peroxidase Substrate Kit (KPL) according to the manufacturer's protocol. For Western Blotting, bound proteins were solubilized in Laemmli buffer.

\section{Scanning for intensely fluorescent targets (SIFT)}

The fluorescent labeling of proteins, incubation for protein aggregation and confocal single-particle analysis were essentially performed as previously described [28]. Prior to the assay, labeled proteins were centrifuged at $100,000 \mathrm{~g}$ for $1 \mathrm{~h}$ at $4^{\circ} \mathrm{C}$ to remove preformed aggregates. Protein concentrations used for aggregation experiments were $64 \mathrm{nM}$ (LTBP-1 $\Delta \mathrm{C}_{-}$V5 and LTBP-1 $1 \Delta \mathrm{N} \_$V5) and $160 \mathrm{nM}$ (N3EGF1-5 WT and N3EGF1-5 R183C). SIFT measurements were performed after $24 \mathrm{~h}$ on an Insight II Reader (Evotec-Technologies).

\section{Statistical analysis}

Data are expressed as mean and standard error of the mean (SEM) of the indicated number of experiments. Statistical analysis was performed using the Sigma Plot 12.5 software and the Mann-Whitney Test.

\section{Results}

\section{Fibronectin and fibrillin-1 do not co-localize with Notch3-ECD deposits}

To study potential molecular mechanisms involved in mediating the toxicity of Notch3-ECD deposits post-mortem brain material from five CADASIL patients and four age- and sex-matched controls was analyzed by different experimental approaches (Table 1). Immunohistochemical staining of Notch3-ECD on paraffin-embedded sections confirmed elevated immunoreactivity in the tunica media of patient arterioles demonstrating the presence of Notch3ECD deposits (Figure 1). Next, fibronectin, fibrillin-1 and latent transforming growth factor- $\beta$ (TGF- $\beta$ ) binding protein 1 (LTBP-1), three ECM proteins with a known role in TGF- $\beta$ bioactivation [35], were analyzed. Fibronectin was detected in moderate amounts in all layers of control vessels, but showed a dramatic increase in the thickened tunica adventitia and intima of CADASIL vessels (Figure 1). Similarly, staining of fibrillin-1 was weak in control vessels showing a fibrillar structure within the tunica adventitia, but was markedly elevated in CADASIL vessels resulting in the continuous labeling of a thin layer at the perimeter of the tunica adventitia (Figure 1). Thus, fibronectin as well as fibrillin-1 accumulate in CADASIL vessels, but their localization appears to differ from Notch3-ECD. The lack of spatial overlap was further demonstrated by immunofluorescence co-staining of frozen patient brain sections for fibrillin-1 and Notch3-ECD (a similar analysis for fibronectin was not possible due to the lack of a suitable antibody). While Notch3-ECD was exclusively detected as granular deposits typical for 


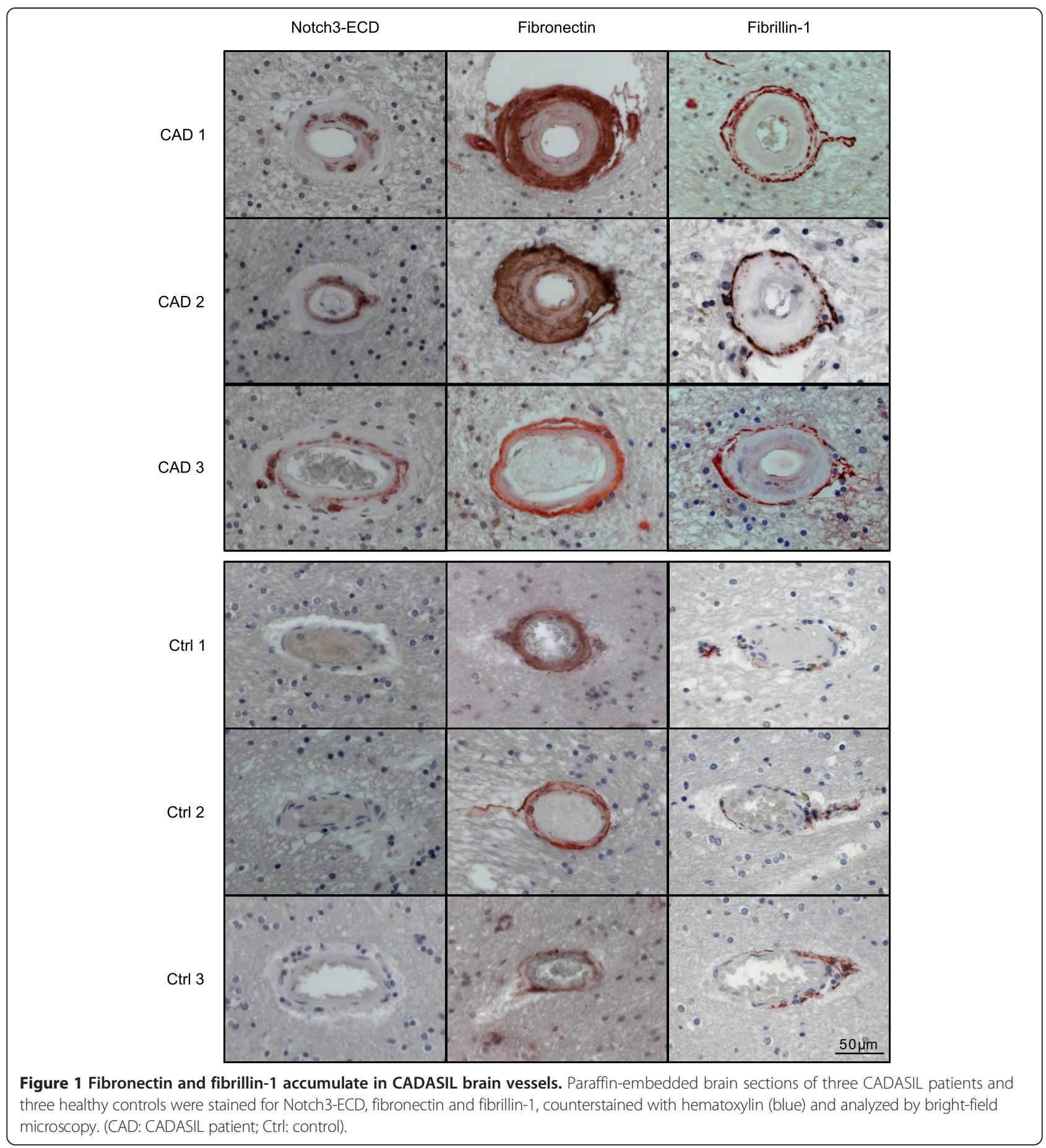

CADASIL vessels [12], fibrillin-1 immunoreactivity again showed a fibrous pattern not overlapping with Notch3ECD staining (Figure 2). These data suggested that fibrillin-1 and fibronectin accumulation in CADASIL vessels is not a result of recruitment into Notch3-ECD deposits, but rather a consequence of fibrotic processes. We, therefore, excluded both proteins as direct participants in Notch3-ECD aggregation.

\section{LTBP-1 is recruited to Notch3-ECD deposits}

Members of the latent TGF- $\beta$ binding protein (LTBP) family, in particular LTBP-1, are key regulators of TGF- $\beta$ bioavailability within the ECM [38] and were found to co-fractionate with Notch3-ECD deposits [30]. We selected LTBP-1, the most prominent member of this family, for analysis and performed immunofluorescence staining on frozen brain sections. In control samples a 


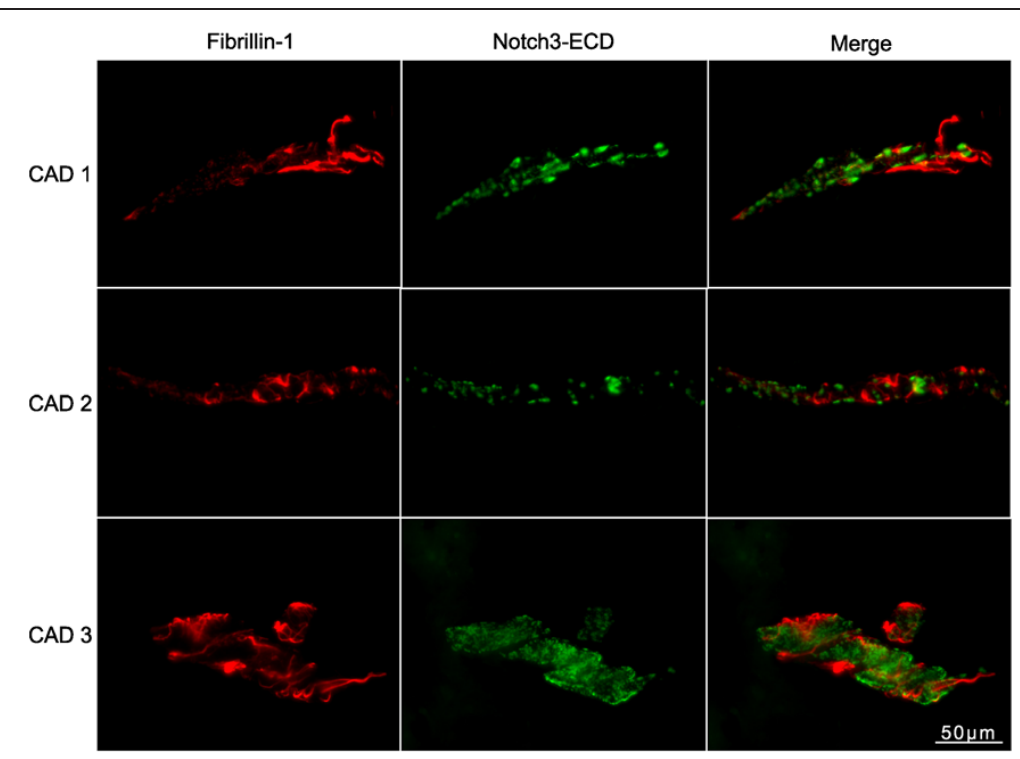

Figure 2 Fibrillin-1 and Notch3-ECD do not co-localize. Frozen brain sections from three CADASIL patients were co-stained for fibrillin-1 and Notch3-ECD and analyzed by fluorescence microscopy. (CAD: CADASIL patient).

weak immunoreactivity in brain arterioles and capillaries was observed (Figure 3a). In contrast, staining was markedly increased in CADASIL vessels and moreover restricted to granules reminiscent of Notch3-ECD deposits, as most clearly seen in capillaries. Indeed, co-staining with Notch3-ECD yielded almost completely overlapping signals in all patients examined (Figure 3b) suggesting specific LTBP-1 recruitment into Notch3-ECD deposits. To verify this finding by an alternative method, we performed biochemical enrichment of Notch3-ECD from isolated brain vessels using sequential protein extraction based on a protocol established previously [30]. Immunoblotting of the final ( $\beta$-MEcontaining) fraction for Notch3-ECD confirmed its enrichment in CADASIL but not control vessels (Figure 3c). Importantly, LTBP-1 showed a similar fractionation behavior further suggesting an association with Notch3-ECD (Figure 3c).

\section{Latency-associated peptide (LAP) accumulates in the tunica media of CADASIL vessels}

The major biological function of LTBP-1 is the sequestration of latent TGF- $\beta$ within the ECM by covalent interaction with latency-associated peptide (LAP) $[38,39]$. We therefore analyzed LAP levels by immunoblotting of the $\beta$-ME fraction of purified brain vessels (Figure 4a) as well as by immunohistochemical staining of paraffin sections (Figure 4b) and detected a clear accumulation in patient, but not control samples. Most importantly, LAP immunoreactivity was restricted to the tunica media (Figure 4b), the vessel layer containing Notch3-ECD deposits. Due to the lack of a suitable antibody, co- localization studies could not be performed. Nonetheless, our findings suggest an LTBP-1-mediated sequestration of LAP into Notch3-ECD aggregates and point to an involvement of the TGF- $\beta$ pathway in CADASIL pathogenesis.

\section{LTBP-1 directly interacts with Notch3-ECD in vitro via its N-terminus}

Since recruitment of ECM proteins into Notch3-ECD deposits might be facilitated by direct protein-protein interactions [30], we investigated binding of LTBP-1 to Notch3-ECD by applying a solid-phase binding assay, an approach successfully used in the past to analyze interaction partners of Notch3 [29] or LTBP-1 [40]. Due to the difficulty to purify correctly folded full-length Notch3-ECD [28], a recombinantly generated Notch3-ECD fragment comprising EGF-like repeats 1-11 and an IgG-Fc affinity tag (N3EGF1-11-Fc) was used. Equally efficient immobilization of N3EGF1-11-Fc and the control ligand IgG-Fc to microtiter plate wells was demonstrated by Western Blotting (Additional file 1: Figure S1). LTBP-1 was provided in soluble form by using conditioned supernatants of transfected HEK293T cells. The amount of LTBP-1 bound to N3EGF1$11-\mathrm{Fc}$ was almost 5-fold increased in comparison to the control ligand (Figure 5a). This was verified by SDS-PAGE and immunoblotting of the material recovered from microtiter plates after assay completion revealing a prominent high-molecular-weight band most likely representing oligomeric LTBP-1 not dissolved under the solubilization conditions used (Figure 5a). These findings suggested a direct interaction between LTBP-1 and the first 11 EGFlike repeats of the Notch3-ECD. To map the binding domain within LTBP-1, two deletion constructs lacking 


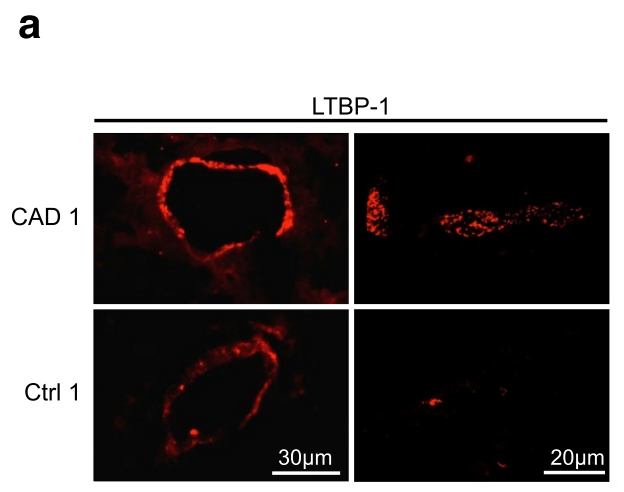

\section{C}

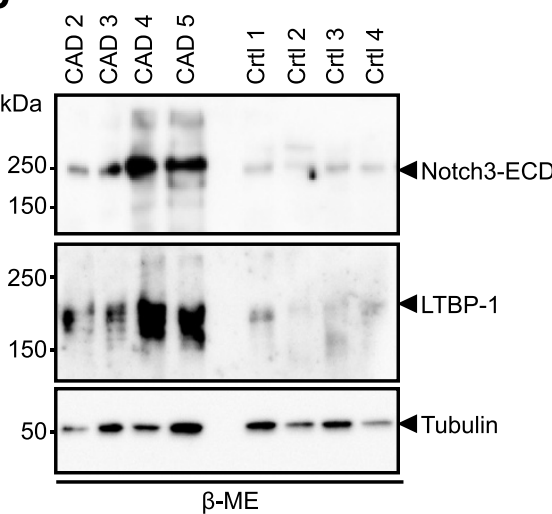

b

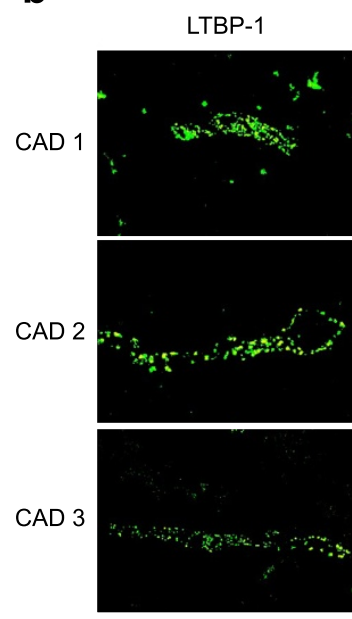

Notch3-ECD
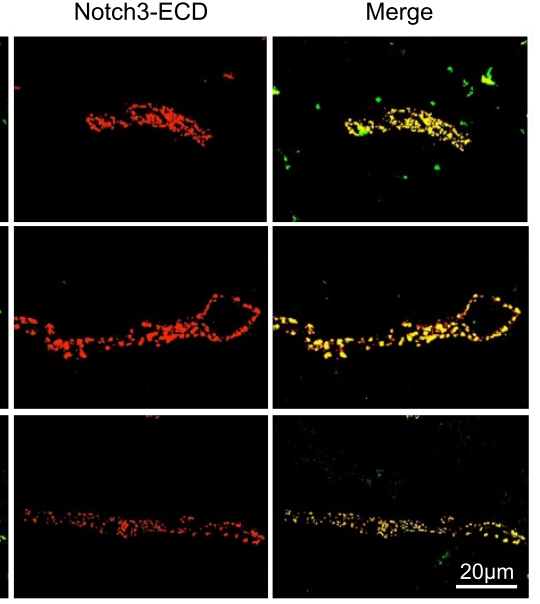

Figure 3 LTBP-1 accumulates in CADASIL brain vessels and co-localizes with Notch3-ECD aggregates. a Frozen brain sections from a CADASIL patient and a healthy control containing an arteriole (left panels) or a capillary (right panels) were stained for LTBP-1 and analyzed by fluorescence microscopy. $\mathbf{b}$ Frozen brain sections of three CADASIL patients were stained for LTBP-1 and Notch3-ECD and analyzed by confocal fluorescence microscopy. c LTBP-1 co-fractionates with Notch3-ECD. The final ( $\beta$-ME-containing) fractions of sequentially extracted brain vessels from CADASIL patients and controls were immunoblotted for Notch3-ECD, LTBP-1 and $\beta$-tubulin. Shown is a representative blot from two independent experiments. (CAD: CADASIL patient; Ctrl: control).

either the $\mathrm{N}$-terminus with the fibronectin interaction domain (LTBP-1 $\triangle \mathrm{N}$ ) or the C-terminus containing the LAP binding site (LTBP-1 $\Delta$ C) were generated (Figure $5 b$ ). Analysis of conditioned supernatants from HEK293T cells overexpressing either one of these constructs revealed significant binding only for LTBP- $1 \Delta C$ (Figure $5 c$ ). To exclude an indirect interaction mediated by other factors present in the supernatant, the binding assay was performed with LTBP- $1 \Delta$ C affinity-purified via its C-terminal His tag (see Figure $6 \mathrm{~b}$ ) and revealed a binding capacity similar to LTBP-1 $\Delta \mathrm{C}$-containing supernatant (Figure $5 \mathrm{c}$ ). This finding suggested that the sequestration of LTBP-1 into Notch3-ECD deposits is mediated by a direct interaction involving its $\mathrm{N}$-terminal region.

\section{LTBP-1 co-aggregates with mutant Notch3}

To provide proof for a direct participation of LTBP-1 in the Notch3-ECD aggregation process, a previously established in vitro assay recapitulating the multimerization of mutant Notch3 was used [27,28]. It is based on scanning for intensely fluorescent targets (SIFT), a unique technique allowing the quantitation of de novo protein aggregation in solution [26]. Purified proteins are labeled with different fluorescent dyes and pairwise incubated to allow the formation of multimeric particles whose size and relative composition is quantified by SIFT and presented in a two-dimensional plot as depicted in Figure 6a. While monomers and lowmolecular-weight oligomers are shown as low-intensity signals, homomeric and heteromeric multimers are represented as mono-colored or dual-colored high-intensity signals, respectively.

For our analyses we used the previously described wildtype and R183C mutant-carrying Notch3 fragments encompassing EGF-like repeats 1-5 (N3EGF1-5) [28] as well as the LTBP-1 deletion variants LTBP- $1 \triangle \mathrm{N}$ and LTBP- 


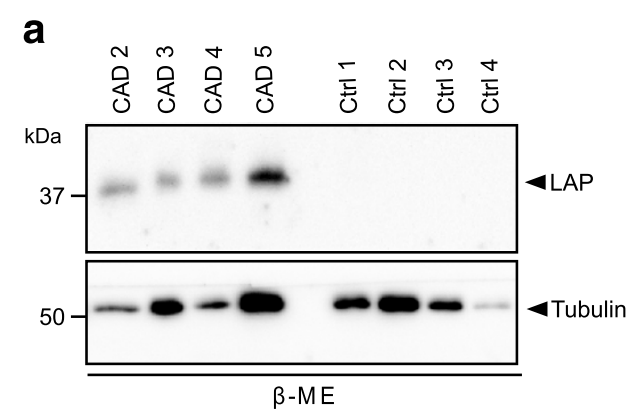

b
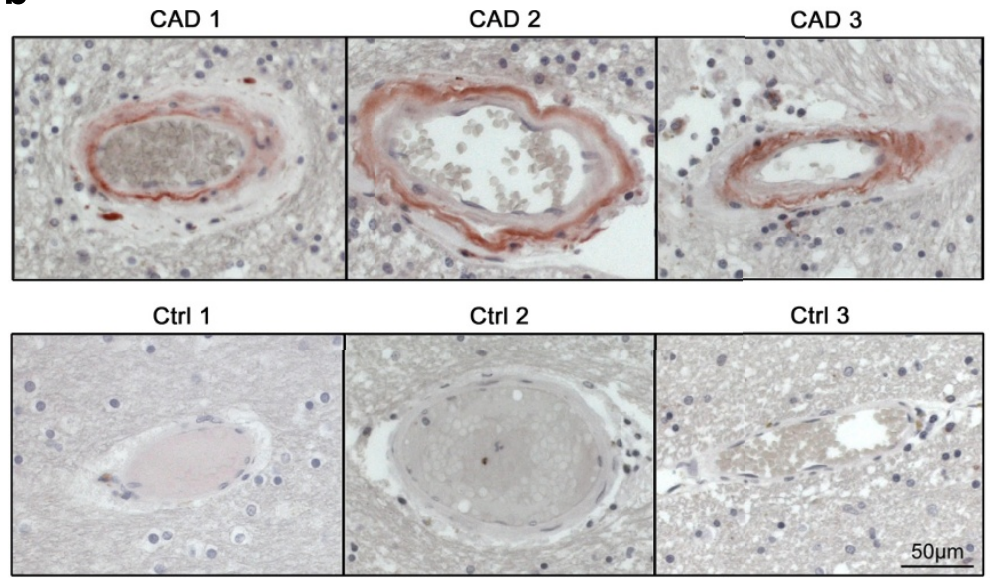

Figure 4 LAP accumulates in CADASIL brain vessels. a LAP co-fractionates with Notch3-ECD. The $\beta$-ME fractions of sequentially extracted brain vessels from CADASIL patients and controls were immunoblotted for LAP and $\beta$-tubulin. Shown is a representative image of two independent extractions. $\mathbf{b}$ LAP accumulates in the tuncia media of CADASIL patient vessels. Paraffin-embedded brain sections were stained for LAP and counterstained with hematoxylin. (CAD: CADASIL patient; Ctrl: control).

$1 \Delta \mathrm{C}$. Figure $6 \mathrm{~b}$ shows the elution fractions of the respective purification procedures used for fluorescent labeling (purity of N3EGF1-5 wild-type was comparable to the R183C mutant, data not shown). In line with our published data, N3EGF1-5 R183C formed higher-order dual-color multimers, while the corresponding wild-type fragment did not show this tendency (Figure $6 \mathrm{c}$ ). When LTBP-1 $\Delta$ C or LTBP$1 \triangle \mathrm{N}$ were analyzed in combination with wild-type N3EGF1-5, only single-color LTBP-1 multimers were detected indicating self-aggregation, a finding already observed in the solid-phase binding assay (see Figure 5a). Predominantly Notch3-containing multimers were obtained when N3EGF1-5 R183C was combined with LTBP-1 $\Delta \mathrm{N}$ lacking the Notch3 interaction domain (Figure 6c). In contrast, the combination of N3EGF1-5 R183C and LTBP-1 $\Delta C$ yielded dual-color multimers with a distribution typical for mutant Notch3 fragments suggesting the generation of aggregates containing both proteins. In summary, these findings provide further support for our hypothesis that LTBP-1 is a component of Notch3-ECD deposits.

\section{Discussion}

The accumulation and deposition of Notch3-ECD, an early manifestation and hallmark of CADASIL [16], is considered the starting point of a chain of pathological events eventually causing brain vessel dysfunction [9]. Motivated by recent evidence implicating the TGF- $\beta$ pathway in sporadic and familial SVD $[32,33]$ and by the identification of fibronectin, fibrillin-1 and members of the LTBP family in brain fractions enriched for Notch3 deposits [30], we studied the role of these ECM constituents in CADASIL-related Notch3-ECD aggregation. For fibronectin and fibrillin-1, fibril-forming glycoproteins required for the LTBP-mediated sequestration of latent TGF- $\beta$ [35], we observe an enrichment in the tunica intima and tunica adventitia of brain vessels from CADASIL patients. A similar increase in fibronectin has previously been reported in a single CADASIL case [41]. However, we do not detect co-localization with Notch3ECD deposits in the tunica media. This finding argues against a direct involvement of fibronectin and fibrillin-1 in Notch3-ECD aggregation, but rather suggests that their accumulation is due to a fibrotic process secondary to Notch3-ECD deposition as previously reported for various types of collagens $[5,6]$.

In contrast, for LTBP-1, the most prominent member of the LTBP family of TGF- $\beta$ signaling regulators [38], we find both an accumulation and a striking co- 


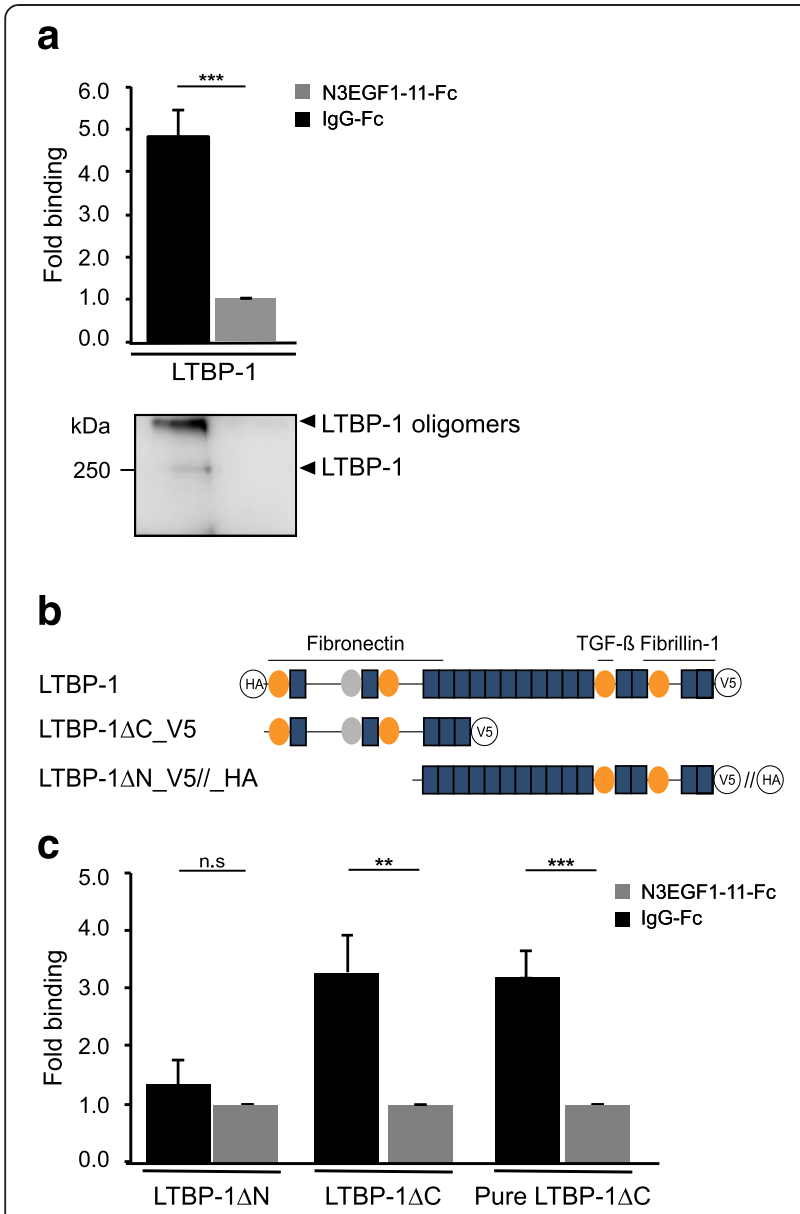

Figure 5 LTBP-1 binds to immobilized Notch3 in a solid-phase binding assay. a The interaction of full-length LTBP-1 derived from conditioned cell supernatants with an immobilized Notch3 fragment (N3EGF1-11-Fc) is increased 4.9-fold when compared to a control ligand (IgG-Fc). Results are expressed as mean + SEM of seven independent experiments. Bound LTBP-1 was re-evaluated by immunoblotting and predominantly detected in oligomeric form. b Schematic representation of the used LTBP-1 constructs and their domain organization including cysteine-rich repeats (orange ovals), a hybrid domain (grey ovals), EGF-like repeats (blue boxes) and V5-His or HA tags (black circles). The fibronectin, TGF- $\beta$, and fibrillin-1 binding regions are indicated. Note that $L T B P-\triangle C-H A$ was used in assays with cell supernatants and LTBP- $\Delta C-V 5$ in assays requiring purified LTBP-1. c LTBP-1 binding to Notch3 is mediated by its $\mathrm{N}$-terminus. While the $\mathrm{N}$-terminal deletion variant LTBP- $1 \Delta \mathrm{N}$ does not bind significantly, the C-terminal deletion variant LTBP- $1 \Delta C$, either from conditioned supernatant or in purified form, shows significant interaction. Results are expressed as mean + SEM of five (LTBP-1 $\left.1 \Delta C_{-} V 5\right)$ and four (LTBP-1 $\left.\triangle \mathrm{N} \_\mathrm{HA}\right)$ independent experiments. n.s.: not significant, ${ }^{* * *} \mathrm{p}<0.001 ;{ }^{* *} \mathrm{p}<0.01$; Mann-Whitney Test.

localization with Notch3-ECD deposits. The presence of this finding in three unrelated patients carrying different NOTCH3 mutations argued for its specificity and prompted us to investigate the relationship between LTBP1 and Notch3-ECD in more detail. By applying a solidphase binding assay we detected a previously unknown direct interaction between the LTBP-1 N-terminal domain and the first 11 EGF-like repeats of Notch3-ECD. This assay relied on the efficient immobilization of the Notch3 protein which could not be achieved with our Notch3ECD fragments encompassing EGF repeats $1-5$ preventing a more precise mapping of the binding site. Likewise, the effect of CADASIL mutations on the LTBP-1-Notch3 interaction could not be investigated since a mutant EGF1-11 fragment was not available. Therefore, mutantspecific effects were studied in a previously established Notch3 aggregation assay $[27,28]$. While the LTBP-1 deletion variant containing the Notch3 binding site showed strong co-aggregation with the archetypal Notch3 mutant $\mathrm{R} 183 \mathrm{C}$, this tendency was not observed with the variant lacking the Notch3 interaction domain. Altogether, these results strongly suggest the recruitment of LTBP-1 into Notch3-ECD deposits by a co-aggregation mechanism requiring a direct interaction.

Our observations extend previous results implicating TIMP-3 and vitronectin in Notch3-ECD deposit formation [30]. In this study, also LTBP-2 and LTBP-4 had been identified by mass spectrometry in human and murine Notch3-ECD preparations, respectively, supporting our data on LTBP-1. The failure to detect it by the proteomic approach might be due to the fact that, instead of isolated vessels, whole brain tissue was used possibly containing minute amounts of LTBP-1. Alternatively, it might be generally difficult to detect LTBPs by mass spectrometry, a possibility supported by the low number of specific peptides assigned to LTBP-2 and LTBP-4 [30]. LTBP-2 does not bind to TGF- $\beta$ [42] and was thus not investigated in our study. We attempted to analyze LTBP-4 in our autopsy material and observed a tendency towards enrichment in patient samples by immunoblotting of $\beta$-ME fractions. However, immunohistological analyses could not be interpreted due to unclear antibody specificity. Thus, further studies are required to assess the role of the individual LTBP family members in the Notch3-ECD aggregation process.

TGF- $\beta$, a well-described regulator of blood vessel formation and homeostasis [43], is secreted from cells as inactive complex consisting of the mature ligand, the TGF- $\beta$ prodomain (also called latent TGF- $\beta$ associated peptide, LAP) and a member of the LTBP family. Sequestration within the ECM is mediated by covalent interactions between LTBPs, fibronectin and fibrillins resulting in the generation of a reservoir of inactive TGF- $\beta$, from which the mature ligand can be released by a variety of mechanisms including proteolysis [35,38]. In agreement with this, we find increased levels of LAP in the tunica media of CADASIL vessels indicating a dysregulation of TGF- $\beta$ signaling. Although co-localization studies with Notch3-ECD could not be performed, our data provide evidence for an involvement of the TGF- $\beta$ pathway in CADASIL pathogenesis. 

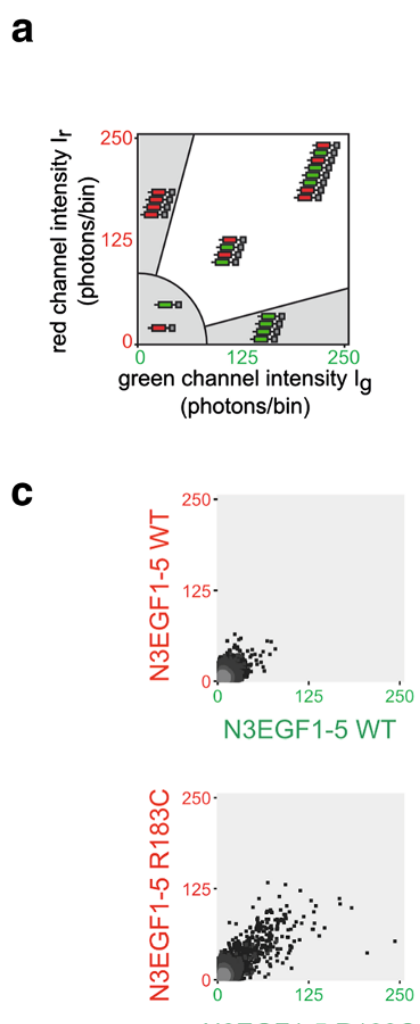

N3EGF1-5 R183C b
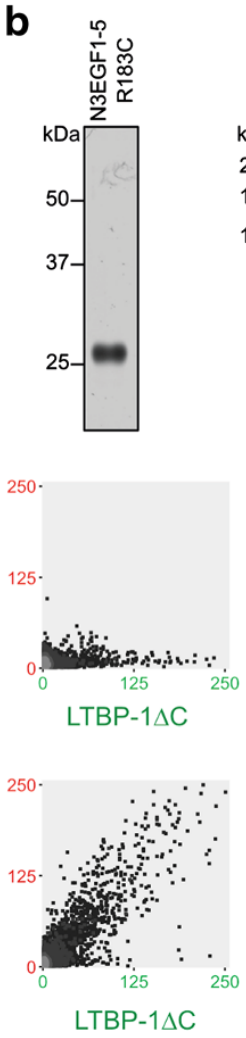
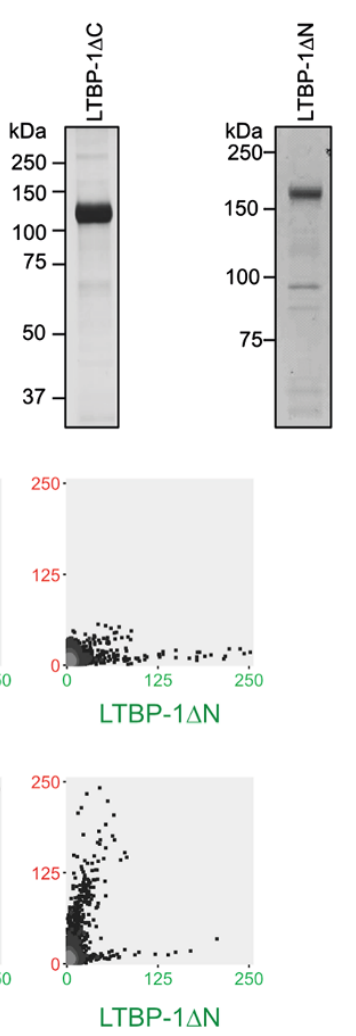

Figure 6 LTBP-1 $1 \Delta$ C specifically co-aggregates with mutant Notch3. a Notch3-ECD multimer formation is monitored by SIFT and data illustrated in a 2D histogram: axes represent the intensity of photons per bin in the detector channel (green channel along the $x$-axis, red channel along the $y$-axis). While monomers and homomeric multimers result in data points in the lower left histogram area and along the axes respectively, heteromeric multimers are represented as high-intensity, dual-color signals in the white sector. $\mathbf{b}$ Purity of proteins used in the aggregation assay. Shown are silver-stained gels containing the elution fractions after metal-ion matrix (LTBP-1 $\Delta \mathrm{C}$ or LTBP-1 $\Delta \mathrm{N}$ ) or Halo-tag-mediated purification (N3EGF1-5 R183C). c SIFT data from different protein combinations: while no high-molecular-weight particles are detected with N3EGF1-5 WT, typical aggregates are formed by N3EGF1-5 R183C and when combined with LTBP-1 $\Delta$ C. In all other combinations homomeric multimers are detected indicating self-aggregation. Shown are representative images of 2-5 experiments. WT: wild-type.

Dysregulated TGF- $\beta$ signaling has been demonstrated in a variety of inherited diseases of the vasculature including Marfan syndrome, an autosomal dominant systemic connective tissue disorder primarily associated with manifestations in the cardiovascular, skeletal and ocular systems [44]. It is caused by mutations in fibrillin-1 which lead to compromised microfibril function, reduced TGF- $\beta$ sequestration and increased TGF- $\beta$ signaling activity. Elevated TGF- $\beta$ levels have also been reported in CARASIL, a rare recessively inherited form of SVD sharing several features with CADASIL [45]. It is caused by loss-of function mutations in the gene encoding high temperature requirement protein A1 (HtrA1), a conserved serine protease with a putative role in the TGF- $\beta$ pathway [33]. CARASIL mutations have been reported to prevent processing of pathway components and to result in elevated TGF- $\beta$ signaling. Thus, it is tempting to speculate that a dysregulation of TGF- $\beta$ activity might represent a common feature of both
CADASIL and CARASIL. More detailed analyses of the different aspects of the TGF- $\beta$ pathway in both diseases are required to substantiate its role in inherited SVD.

\section{Additional file}

Additional file 1: Figure S1. N3EGF1-11-Fc and IgG-Fc display similar immobilization efficiency. Bound proteins were recovered from wells by the addition of Laemmli buffer after assay completion and compared to $100 \mathrm{ng}$ purified protein. N3EGF1-11-FC and IgG-Fc were immunodetected using an anti-human-HRP antibody.

\section{Competing interests}

The authors declare that they have no competing interests.

\section{Authors' contributions}

JK, CO, CH designed research; JK, PH performed research; NB, AJ, AG contributed reagents/analytic tools; JK, CH analyzed data; JK, MD, CO, CH wrote the paper. All authors read and approved the final manuscript. 


\section{Acknowledgements}

We are grateful to Thomas Arzberger (Brain-Net, LMU, Munich) for kindly providing the human brain samples, Elisabeth Kremmer (Helmholtz Center, Munich) for generating anti-Notch3-ECD antibodies and Jorma Keski-Oja (University of Helsinki, Finland) for providing LTBP-1 plasmids. We thank Eva Scharrer and Marco Düring (ISD, Munich) for help with the brain vessel isolation procedure and preparation of SIFT figures.

This work was supported by the Dr. Werner Jackstädt-Stiftung (to C.O.), the Fondation Leducq (Transatlantic Network of Excellence on the Pathogenesis of Small Vessel Disease of the Brain) (to A.J. and M.D.) and the Vascular Dementia Research Foundation (to M.D.).

\section{Author details}

${ }^{1}$ Institute for Stroke and Dementia Research, Klinikum der Universität München, Ludwig-Maximilians University Munich, Max-Lebsche-Platz 30, 81377 Munich, Germany. ${ }^{2}$ Munich Center for Systems Neurology (SyNergy), Schillerstr. 44, 80336 Munich, Germany. ${ }^{3}$ Center for Neuropathology and Prion Research, Ludwig-Maximilians University Munich, Feodor-Lynen-Str. 23, 81377 Munich, Germany. ${ }^{4}$ INSERM, U1161, F-75010 Paris, France. ${ }^{5}$ Univ Paris Diderot, Sorbonne Paris Cité, 10 av de Verdun, UMRS 1161, F-75010 Paris, France.

\section{Received: 26 June 2014 Accepted: 4 August 2014}

Published: 13 August 2014

\section{References}

1. Pantoni $L$ (2010) Cerebral small vessel disease: from pathogenesis and clinical characteristics to therapeutic challenges. Lancet Neurol 9(7):689-701, doi:10.1016/s1474-4422(10)70104-6

2. Wardlaw JM, Smith C, Dichgans M (2013) Mechanisms of sporadic cerebral small vessel disease: insights from neuroimaging. Lancet Neurol 12(5):483-497, doi:10.1016/s1474-4422(13)70060-7

3. Dichgans M (2007) Genetics of ischaemic stroke. Lancet Neurol 6(2):149-161, doi:10.1016/s1474-4422(07)70028-5

4. Yamamoto Y, Craggs L, Baumann M, Kalimo H, Kalaria RN (2011) Review: molecular genetics and pathology of hereditary small vessel diseases of the brain. Neuropathol Appl Neurobiol 37(1):94-113, doi:10.1111/j.13652990.2010.01147.x

5. Miao Q, Paloneva T, Tuominen S, Poyhonen M, Tuisku S, Viitanen M, Kalimo $H$ (2004) Fibrosis and stenosis of the long penetrating cerebral arteries: the cause of the white matter pathology in cerebral autosomal dominant arteriopathy with subcortical infarcts and leukoencephalopathy. Brain Pathol 14(4):358-364, doi:10.1111/j.1750-3639.2004.tb00078

6. Dong H, Blaivas M, Wang MM (2012) Bidirectional encroachment of collagen into the tunica media in cerebral autosomal dominant arteriopathy with subcortical infarcts and leukoencephalopathy. Brain Res 1456:64-71, doi:10.1016/j.brainres.2012.03.037

7. Joutel A, Faraci FM (2014) Cerebral small vessel disease: insights and opportunities from mouse models of collagen IV-related small vessel disease and cerebral autosomal dominant arteriopathy with subcortical infarcts and leukoencephalopathy. Stroke 45(4):1215-1221, doi:10.1161/strokeaha. 113.002878

8. Chabriat $H$, Joutel A, Dichgans M, Tournier-Lasserve E, Bousser MG (2009) CADASIL. Lancet Neurol 8(7):643-653, doi:10.1016/S1474-4422(09)70127-9

9. Joutel A (2011) Pathogenesis of CADASIL: transgenic and knock-out mice to probe function and dysfunction of the mutated gene, Notch3, in the cerebrovasculature. BioEssays 33(1):73-80, doi:10.1002/bies.201000093

10. Joutel A, Corpechot C, Ducros A, Vahedi K, Chabriat H, Mouton P, Alamowitch S, Domenga V, Cecillion M, Marechal E, Maciazek J, Vayssiere C, Cruaud C, Cabanis EA, Ruchoux MM, Weissenbach J, Bach JF, Bousser MG, Tournier-Lasserve E (1996) Notch3 mutations in CADASIL a hereditary adultonset condition causing stroke and dementia. Nature 383(6602):707-10, doi:10.1038/383707a0

11. Peters N, Opherk C, Bergmann T, Castro M, Herzog J, Dichgans M (2005) Spectrum of mutations in biopsy-proven CADASIL: implications for diagnostic strategies. Arch Neurol 62(7):1091-1094, doi:10.1001/archneur.62.7.1091

12. Joutel A, Andreux F, Gaulis S, Domenga V, Cecillon M, Battail N, Piga N, Chapon F, Godfrain C, Tournier-Lasserve E (2000) The ectodomain of the Notch3 receptor accumulates within the cerebrovasculature of CADASIL patients. J Clin Invest 105(5):597-605, doi:10.1172/JC18047
13. Ishiko A, Shimizu A, Nagata E, Takahashi K, Tabira T, Suzuki N (2006) Notch3 ectodomain is a major component of granular osmiophilic material (GOM) in CADASIL. Acta Neuropathol 112(3):333-339, doi:10.1007/s00401-006-0116-2

14. Tikka S, Mykkanen $K$, Ruchoux MM, Bergholm R, Junna M, Poyhonen M, Yki-Jarvinen $H$, Joutel A, Viitanen M, Baumann M, Kalimo H (2009) Congruence between NOTCH3 mutations and GOM in 131 CADASIL patients. J Neurol 132(Pt 4):933-939, doi:10.1093/brain/awn364

15. Yamamoto Y, Craggs LJ, Watanabe A, Booth T, Attems J, Low RW, Oakley AE, Kalaria RN (2013) Brain microvascular accumulation and distribution of the NOTCH3 ectodomain and granular osmiophilic material in CADASIL. J Neuropathol Exp Neurol 72(5):416-431, doi:10.1097/NEN.0b013e31829020b5

16. Mayer M, Straube A, Bruening R, Uttner I, Pongratz D, Gasser T, Dichgans M, Muller-Hocker J (1999) Muscle and skin biopsies are a sensitive diagnostic tool in the diagnosis of CADASIL. J Neurol 246(7):526-532, doi:10.1007/ s004150050398

17. Lesnik Oberstein SA, van Duinen SG, van den Boom R, Maat-Schieman ML, van Buchem MA, van Houwelingen HC, Hegeman-Kleinn IM, Ferrari MD, Breuning MH, Haan J (2003) Evaluation of diagnostic NOTCH3 immunostaining in CADASIL. Acta Neuropathol 106(2):107-111, doi:10.1007/s00401-003-0701-6

18. Monet M, Domenga V, Lemaire B, Souilhol C, Langa F, Babinet C, Gridley T, Tournier-Lasserve E, Cohen-Tannoudji M, Joutel A (2007) The archetypal R90C CADASIL-NOTCH3 mutation retains NOTCH3 function in vivo. Hum Mol Genet 16(8):982-992, doi:10.1093/hmg/ddm042

19. Monet-Lepretre M, Bardot B, Lemaire B, Domenga V, Godin O, Dichgans M, Tournier-Lasserve E, Cohen-Tannoudji M, Chabriat H, Joutel A (2009) Distinct phenotypic and functional features of CADASIL mutations in the Notch3 ligand binding domain. Brain 132(6):1601, doi:10.1093/brain/awp049

20. Joutel A, Monet-Lepretre M, Gosele C, Baron-Menguy C, Hammes A, Schmidt S, Lemaire-Carrette B, Domenga V, Schedl A, Lacombe P, Hubner N (2010) Cerebrovascular dysfunction and microcirculation rarefaction precede white matter lesions in a mouse genetic model of cerebral ischemic small vessel disease. J Clin Invest 120(2):433-445, doi:10.1172/JCI39733

21. Karlstrom H, Beatus P, Dannaeus K, Chapman G, Lendahl U, Lundkvist J (2002) A CADASIL-mutated Notch 3 receptor exhibits impaired intracellular trafficking and maturation but normal ligand-induced signaling. Proc Natl Acad Sci U S A 99(26):17119-17124, doi:10.1073/pnas.252624099

22. Low WC, Santa Y, Takahashi K, Tabira T, Kalaria RN (2006) CADASIL-causing mutations do not alter Notch3 receptor processing and activation. Neuroreport 17(10):945-949, doi:10.1097/01.wnr.0000223394.66951.48

23. Peters N, Opherk C, Zacherle S, Capell A, Gempel P, Dichgans M (2004) CADASIL-associated Notch3 mutations have differential effects both on ligand binding and ligand-induced Notch3 receptor signaling through RBP-Jk. Exp Cell Res 299(2):454-464, doi:10.1016/j.yexcr.2004.06.004

24. Arboleda-Velasquez JF, Manent J, Lee JH, Tikka S, Ospina C, Vanderburg CR, Frosch MP, Rodriguez-Falcon M, Villen J, Gygi S, Lopera F, Kalimo H, Moskowitz MA, Ayata C, Louvi A, Artavanis-Tsakonas S (2011) Hypomorphic Notch 3 alleles link Notch signaling to ischemic cerebral small-vessel disease. Proc Natl Acad Sci U S A 108(21):E128-E135, doi:10.1073/pnas.1101964108

25. Rutten JW, Boon EM, Liem MK, Dauwerse JG, Pont MJ, Vollebregt E, Maat-Kievit AJ, Ginjaar HB, Lakeman P, van Duinen SG, Terwindt GM, Lesnik Oberstein SA (2013) Hypomorphic NOTCH3 alleles do not cause CADASIL in humans. Hum Mutat 34(11):1486-1489, doi:10.1002/humu.22432

26. Giese A, Bader B, Bieschke J, Schaffar G, Odoy S, Kahle PJ, Haass C, Kretzschmar $\mathrm{H}$ (2005) Single particle detection and characterization of synuclein co-aggregation. Biochem Biophys Res Commun 333(4):1202-1210, doi:10.1016/j.bbrc.2005.06.025

27. Opherk C, Duering M, Peters N, Karpinska A, Rosner S, Schneider E, Bader B, Giese A, Dichgans M (2009) CADASIL mutations enhance spontaneous multimerization of NOTCH3. Hum Mol Genet 18(15):2761-2767, doi:10.1093/ $\mathrm{hmg} / \mathrm{ddp} 211$

28. Duering M, Karpinska A, Rosner S, Hopfner F, Zechmeister M, Peters N, Kremmer E, Haffner C, Giese A, Dichgans M, Opherk C (2011) Co-aggregate formation of CADASIL-mutant NOTCH3: a single-particle analysis. Hum Mol Genet 20(16):3256-3265, doi:10.1093/hmg/ddr237

29. Meng H, Zhang $X$, Hankenson KD, Wang MM (2009) Thrombospondin 2 potentiates notch3/jagged1 signaling. J Biol Chem 284(12):7866-7874, doi:10.1074/jbc.M803650200

30. Monet-Lepretre M, Haddad I, Baron-Menguy C, Fouillot-Panchal M, Riani M, Domenga-Denier V, Dussaule C, Cognat E, Vinh J, Joutel A (2013) Abnormal recruitment of extracellular matrix proteins by excess Notch3 ECD: a new pathomechanism in CADASIL. Brain 136(6):1830, doi:10.1093/brain/awt092 
31. Ruiz-Ortega M, Rodríguez-Vita J, Sanchez-Lopez E, Carvajal G, Egido J (2007) TGF- $\beta$ signaling in vascular fibrosis. Cardiovasc Res 74(2):196-206, doi:10.1016/j.cardiores.2007.02.008

32. Thompson CS, Hakim AM (2009) Living beyond our physiological means: small vessel disease of the brain is an expression of a systemic failure in arteriolar function: a unifying hypothesis. Stroke 40(5):e322-e330, doi:10.1161/strokeaha.108.542266

33. Hara K, Shiga A, Fukutake T, Nozaki H, Miyashita A, Yokoseki A, Kawata H, Koyama A, Arima K, Takahashi T, Ikeda M, Shiota H, Tamura M, Shimoe Y, Hirayama M, Arisato T, Yanagawa S, Tanaka A, Nakano I, Ikeda S, Yoshida Y, Yamamoto T, Ikeuchi T, Kuwano R, Nishizawa M, Tsuji S, Onodera O (2009) Association of HTRA1 mutations and familial ischemic cerebral small-vessel disease. N Engl J Med 360(17):1729-1739, doi:10.1056/NEJMoa0801560

34. Shiga A, Nozaki H, Yokoseki A, Nihonmatsu M, Kawata H, Kato T, Koyama A Arima K, Ikeda M, Katada S, Toyoshima Y, Takahashi H, Tanaka A, Nakano I, Ikeuchi T, Nishizawa M, Onodera O (2011) Cerebral small-vessel disease protein HTRA1 controls the amount of TGF-beta1 via cleavage of proTGF-beta1. Hum Mol Genet 20(9):1800-1810, doi:10.1093/hmg/ddr063

35. Hynes RO (2009) The extracellular matrix: not just pretty fibrils. Science 326(5957):1216-1219, doi:10.1126/science.1176009

36. Gayraud B, Keene DR, Sakai LY, Ramirez F (2000) New insights into the assembly of extracellular microfibrils from the analysis of the fibrillin 1 mutation in the tight skin mouse. J Cell Biol 150(3):667-680, doi:10.1083/ jcb.150.3.667

37. Saharinen J, Taipale J, Keski-Oja J (1996) Association of the small latent transforming growth factor-beta with an eight cysteine repeat of its binding protein LTBP-1. EMBO 15(2):245-253

38. Todorovic V, Rifkin DB (2012) LTBPs, more than just an escort service. J Cell Biochem 113(2):410-418, doi:10.1002/jcb.23385

39. Shi M, Zhu J, Wang R, Chen X, Mi L, Walz T, Springer TA (2011) Latent TGF-beta structure and activation. Nature 474(7351):343-349, doi:10.1038/ nature 10152

40. Massam-Wu T, Chiu M, Choudhury R, Chaudhry SS, Baldwin AK, McGovern A, Baldock C, Shuttleworth CA, Kielty CM (2010) Assembly of fibrillin microfibrils governs extracellular deposition of latent TGF beta. J Cell Sci 123(Pt 17):3006-3018, doi:10.1242/jcs.073437

41. Oide T, Nakayama H, Yanagawa S, Ito N, Ikeda S, Arima K (2008) Extensive loss of arterial medial smooth muscle cells and mural extracellular matrix in cerebral autosomal recessive arteriopathy with subcortical infarcts and leukoencephalopathy (CARASIL). Neuropathology 28(2):132-142, doi:10.1111/j.1440-1789.2007.00864.x

42. Saharinen J, Keski-Oja J (2000) Specific sequence motif of 8-Cys repeats of TGF-beta binding proteins, LTBPs, creates a hydrophobic interaction surface for binding of small latent TGF-beta. Mol Biol Cell 11(8):2691-2704, doi:10.1091/mbc.11.8.2691

43. Pardali E, Ten Dijke P (2012) TGF-beta signaling and cardiovascular diseases. Int J Biol Sci 8(2):195-213, doi:10.7150/ijbs.3805

44. Doyle JJ, Gerber EE, Dietz HC (2012) Matrix-dependent perturbation of TGF-beta signaling and disease. FEBS Lett 586(14):2003-2015, doi:10.1016/j. febslet.2012.05.027

45. Fukutake T (2011) Cerebral autosomal recessive arteriopathy with subcortical infarcts and leukoencephalopathy (CARASIL): from discovery to gene identification. J Stroke Cerebrovasc Dis 20(2):85-93, doi:10.1016/j. jstrokecerebrovasdis.2010.11.008

doi:10.1186/s40478-014-0096-8

Cite this article as: Kast et al: Sequestration of latent TGF- $\beta$ binding

protein 1 into CADASIL-related Notch3-ECD deposits. Acta

Neuropathologica Communications 2014 2:96.

\section{Submit your next manuscript to BioMed Central and take full advantage of:}

- Convenient online submission

- Thorough peer review

- No space constraints or color figure charges

- Immediate publication on acceptance

- Inclusion in PubMed, CAS, Scopus and Google Scholar

- Research which is freely available for redistribution 